\title{
Influenza vaccination motivators among healthcare personnel in a large acute care hospital in Israel
}

\author{
Amir Nutman ${ }^{*}$ and Naomi Yoeli
}

\begin{abstract}
Background: Vaccinating healthcare personnel (HCP) against influenza is important to prevent transmission and morbidity among patients and staff.

Methods: We conducted an online survey assessing knowledge, perceptions and attitudes concerning influenza vaccination among HCP. Multivariate logistic regression was performed to identify independent predictors of vaccination.

Results: The survey was completed by 468 HCP representing all categories of staff. Doctors believed that vaccination was the best way to prevent influenza and perceived the vaccine less harmful as compared to nurses and allied health professionals. Getting vaccinated was associated with a greater likelihood of recommending vaccination to patients: $86 \%$ vs. $54 \%$ in vaccinated and unvaccinated HCP, respectively. Reasons for vaccine refusal were fear of needles (19\%); fear of side effects (66\%) and lack of time (16\%). In the multivariate analysis, survey items that were independently associated with vaccination were beliefs that: vaccine effectively prevents influenza (OR $4.0795 \% \mathrm{Cl}$ 2.51, 6.58); HCP are at increased risk of influenza (OR $2.8295 \% \mathrm{Cl}$ 1.56, 5.13); vaccine can cause influenza (OR $0.4195 \% \mathrm{Cl} 0.25,0.65)$; contracting influenza is likely in the absence of vaccination (OR $1.9695 \% \mathrm{Cl}$ 1.12, 3.42); and that HCP might transmit influenza to their family (OR $4.5495 \% \mathrm{Cl} 1.38$, 14.97). The belief that HCP might transmit influenza to patients was not independently associated with vaccine uptake.

Conclusion: Our study revealed misconceptions and knowledge gaps concerning the risk of influenza and the influenza vaccine. There were significant differences in knowledge and attitudes between healthcare professions. HCP decline vaccination because they do not perceive a personal risk of influenza infection and are concerned about side effects. Thus, in order to increase vaccination rates it is important to educate HCP to correct misconceptions concerning vaccine efficacy and safety, while promoting the benefit of getting vaccinated in order to protect themselves and their families.
\end{abstract}

Keywords: Influenza vaccine, Knowledge, perceptions and attitudes, Healthcare personnel vaccination uptake, Survey

\footnotetext{
* Correspondence: amirn@tlvmc.gov.il

Tel-Aviv Sourasky Medical Center, 6 Weizmann St., Tel Aviv 64239, Israel
} 


\section{Background}

Healthcare personnel (HCP) are an important priority group for influenza vaccination in order to protect them against influenza infection and to prevent transmission to patients, especially high-risk populations such as immune-compromised and elderly patients [1-3]. Despite this, vaccination coverage of $\mathrm{HCP}$ is suboptimal worldwide $[4,5]$ and in the United States many institutions have successfully implemented a mandatory vaccination policy as a means to increase coverage [6].

In Israel, the Ministry of Health recommends influenza vaccination for all $\mathrm{HCP}$ and set a quality target of $60 \%$ coverage [7]; however, there are no legal means to enforce this policy and vaccination uptake is unsatisfactory [8]. In the 2014-2015 influenza season, the average vaccination rate in acute care hospitals in Israel was $41 \%$ (range 14-78 \%): $47 \%$ among doctors, $35 \%$ among nurses and $37 \%$ among allied health professionals [9].

As part of a program to increase vaccination coverage among HCP at our hospital, we conducted an online survey to assess motivating factors and barriers for immunization, identify target groups for intervention and explore attitudes toward a policy of mandatory vaccination.

\section{Methods}

\section{Setting}

The study was conducted at Tel-Aviv Sourasky Medical Center, a 1450-bed tertiary care academic hospital in Israel. Influenza vaccination is offered free of charge for all clinical and non-clinical personnel every year. In order to make vaccines more accessible, they are administered by mobile nurse teams who come to the clinical units, the personnel clinic and in public areas such as the cafeteria and conferences. Vaccines are also distributed to the wards for vaccinating staff working evening and night shifts. The influenza campaign also consists of educational material (posters, mailings and talks) and hospital leadership involvement.

\section{Data collection}

Information about overall vaccination rate for the 2014-2015 influenza season was collected from employee immunization records.

The online survey was conducted at the end of the flu campaign of 2014-2015. Employees were invited to complete the online survey via an e-mail with a link to the survey. In order to increase participation, the survey was anonymous.

The survey consisted of 30 questions: demographic information including age, gender, profession (doctor, nurse, allied health professional or others including administrative and support staff), and department; knowledge about influenza disease and vaccine; perception of flu risk; perception of vaccine efficacy and side- effects; practices of recommending vaccination to family and patients, and attitude towards a mandatory vaccination policy. Each participant was asked to indicate whether they had been vaccinated in the survey year. Because the survey was anonymous, participant information could not be cross-referenced to immunization records. Participants who indicated that they were not vaccinated were asked about inhibiting factors: fear of vaccine side effects, fear of needles, lack of time and medical exemption from vaccination. Participants who indicated that they were vaccinated were asked about side effects after vaccination.

Answers to survey questions took two forms: agree, disagree or unsure and yes or no. Regarding recommending vaccination to patients, participants were allowed to answer "not relevant" if not engaged in direct patient care.

\section{Data analysis}

We limited our analysis to participants who completed the entire survey. Data were summarized descriptively. For the purpose of analysis, "unsure" was grouped with "disagree". Associations of survey items with profession were analyzed by chi-square tests. Odds ratios and $95 \%$ confidence intervals for vaccination were calculated by simple logistic regression. Multivariate logistic regression, using forward selection of variables, was performed to identify independent predictors of vaccination among HCP. Demographic information and all survey questions were considered for the model. For all tests performed, a two-sided $p$-value $\leq 0.05$ was considered statistically significant.

Data were analyzed using SPSS version 22 (IBM, Armonk, NY).

\section{Results}

\section{Influenza vaccine uptake}

During the 2014-2015 influenza season, the overall influenza vaccination rate at our hospital was $42 \%$ : $56 \%$ among physicians, $41 \%$ among nurses, $37 \%$ among allied health professionals, and $30 \%$ among others (administration and support staff).

\section{Survey participants}

The survey was completed by 468 employees. Demographic characteristics of participants are summarized in Table 1. Among survey participants, 310 (66 \%) indicated they were vaccinated: $102(84 \%)$ of physicians, 60 (65\%) of nurses, 69 (57\%) of allied health professions and 79 (59\%) of others.

\section{Knowledge concerning influenza}

Results of survey questions concerning knowledge about influenza are presented in Table 2: almost all participants agreed that influenza is widespread and can have 
Table 1 Characteristics of study sample $(N=468)$

\begin{tabular}{ll}
\hline Characteristic & $n(\%)$ \\
\hline Sex female & $339(72.4)$ \\
Age category & $195(41.7)$ \\
18-39 years & $258(55.1)$ \\
$40-65$ years & $15(3.2)$ \\
$>65$ years & \\
Profession & $93(19.9)$ \\
Nurses & $121(25.9)$ \\
Doctors & $121(25.9)$ \\
Allied health professionals & $133(28.4)$ \\
Others & \\
Department & $150(32.1)$ \\
Medical & $84(17.9)$ \\
Surgical & $15(3.2)$ \\
Obstetrics \& Gynecology & $52(11.1)$ \\
Pediatrics & $19(4.1)$ \\
Radiology & $32(6.8)$ \\
Laboratories & $116(24.8)$ \\
Other Non-clinical & $310(66.2)$ \\
Vaccinated against influenza &
\end{tabular}

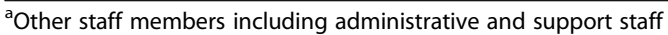

severe complications including death. However, only 385 (82\%) of participants agreed that as HCP they are at increased risk for infection by influenza. This proportion was higher among doctors (94\%) than among nurses and allied health professionals (79 \%). The OR for vaccination was $2.2(95 \%$ CI 1.1, 4.5) for participants who agreed that influenza can have severe complications, and 5.8 (95\% CI 3.5, 9.6) for participants who agreed that they are at increased risk for influenza.

\section{Knowledge concerning influenza vaccine}

Results of survey questions concerning knowledge about vaccine are presented in Table 3: 292 (62 \%) of participants agreed that vaccination is the best way to prevent influenza. This proportion was higher among doctors (89 \%) than among nurses (55\%) and allied health professionals $(49 \%)$. The OR for vaccination was 7.4 (95\% CI 4.8, 11.2) for participants who agreed that vaccination is the best way to prevent influenza. Almost half of participants, 228 (48.7\%), agreed that vaccination can cause influenza. This belief was more common among nurses $(61 \%)$ and allied health professionals $(55 \%)$ than among doctors (22\%). The OR for vaccination was 0.28 (95\% CI 0.19, 0.42) for participants who agreed that vaccine can cause the flu. Only 269 (57.5 \%) disagreed that vaccine side effects can be more severe than the flu; disagreeing was less common among nurses (45\%) and allied health professionals (55\%) than among doctors (81\%). The OR for vaccination was 0.35 (95\% CI 0.22 , 0.55) for participants who agreed that vaccine side effects can be more severe than the flu.

\section{Perceived influenza risk}

Results of survey questions concerning perceived personal risk are presented in Table 4: Only 75 (16\%) agreed that they get sick with the flu easily and only 144 (31 \%) agreed that if they don't get vaccinated they get sick with the flu. There was no significant difference between healthcare professions. The OR for vaccination was $1.6(95 \%$ CI $0.9,2.8)$ for participants who agreed that they catch the flu easily, and 3.5 (95\% CI 2.2, 5.8) for participants who agreed that if they don't get vaccinated they get sick with the flu.

Most of the participants (95\%) agreed that if they get sick with influenza they may infect their family. The proportion of participants who agreed that they may

Table 2 Knowledge concerning influenza disease $(N=468)$

\begin{tabular}{|c|c|c|c|c|c|c|c|}
\hline & All & Nurses & Doctors & Allied health professionals & Others & $p$-value ${ }^{a}$ & OR for vaccination (95\% Cl) \\
\hline \multicolumn{8}{|c|}{ Influenza is widespread and can affect any person at any age } \\
\hline Agree, $n(\%)$ & $459(98.1)$ & $92(98.9)$ & $120(99.2)$ & $120(99.2)$ & $127(95.5)$ & 0.085 & $0.981(0.242,3.974)$ \\
\hline Disagree, $n(\%)$ & $1(0.2)$ & $1(1.1)$ & & & & & Reference \\
\hline Unsure, $n(\%)$ & $8(1.7)$ & & $1(0.8)$ & $1(0.8)$ & $6(4.5)$ & & \\
\hline \multicolumn{8}{|c|}{ Influenza can have severe complications including death } \\
\hline Agree, $n(\%)$ & $435(92.9)$ & $86(92.5)$ & $120(99.2)$ & $110(90.9)$ & $119(89.5)$ & 0.015 & $2.215(1.087,4.513)$ \\
\hline Disagree, $n(\%)$ & $9(1.9)$ & $3(3.2)$ & & $2(1.7)$ & $4(3)$ & & Reference \\
\hline Unsure, $n(\%)$ & $24(5.1)$ & $4(4.3)$ & $1(0.8)$ & $9(7.4)$ & $10(7.5)$ & & \\
\hline \multicolumn{8}{|c|}{ Hospital workers are at increased risk of contracting influenza because of their job } \\
\hline Agree, $n(\%)$ & $385(82.3)$ & $73(78.5)$ & $114(94.2)$ & $96(79.3)$ & $102(76.7)$ & 0.001 & $5.755(3.449,9.602)$ \\
\hline Disagree, $n(\%)$ & $25(5.3)$ & $8(8.6)$ & $1(0.8)$ & $9(7.4)$ & $7(5.3)$ & & Reference \\
\hline Unsure, $n(\%)$ & $58(12.4)$ & $12(12.9)$ & $6(5)$ & $16(13.2)$ & $24(18)$ & & \\
\hline
\end{tabular}

${ }^{a} p$-value for association between survey item and profession 
Table 3 Knowledge concerning influenza vaccine $(N=468)$

\begin{tabular}{|c|c|c|c|c|c|c|c|}
\hline & All & Nurses & Doctors & Allied health professionals & Others & $p$-value ${ }^{a}$ & OR for vaccination (95\% Cl) \\
\hline \multicolumn{8}{|c|}{ The most effective way to prevent influenza is vaccination } \\
\hline Agree, $n(\%)$ & $292(62.4)$ & $51(54.8)$ & $108(89.3)$ & $59(48.8)$ & $74(55.6)$ & \multirow[t]{3}{*}{$<0.001$} & $7.328(4.778,11.238)$ \\
\hline Disagree, $n(\%)$ & $60(12.8)$ & $19(20.4)$ & $3(2.5)$ & $17(14)$ & $21(15.8)$ & & Reference \\
\hline Unsure, $n(\%)$ & $116(24.8)$ & $23(24.7)$ & $10(8.3)$ & $45(37.2)$ & $38(28.6)$ & & \\
\hline \multicolumn{8}{|c|}{ Vaccine side effects can be more severe than the flu } \\
\hline Agree, $n(\%)$ & $93(19.9)$ & $30(32.3)$ & $12(9.9)$ & $23(19)$ & $28(21.1)$ & \multirow[t]{3}{*}{0.001} & $0.348(0.219,0.554)$ \\
\hline Disagree, $n(\%)$ & $269(57.5)$ & $42(45.2)$ & $98(81)$ & $67(55.4)$ & $62(46.6)$ & & Reference \\
\hline Unsure, $n(\%)$ & $106(22.6)$ & $21(22.6)$ & $11(9.1)$ & $31(25.6)$ & $43(32.3)$ & & \\
\hline \multicolumn{8}{|c|}{ Vaccine can cause the flu } \\
\hline Agree, $n(\%)$ & $228(48.7)$ & $57(61.3)$ & $26(21.5)$ & $67(55.4)$ & $78(58.6)$ & \multirow[t]{3}{*}{$<0.001$} & $0.280(0.186,0.421)$ \\
\hline Disagree, $n(\%)$ & $150(32.1)$ & $23(24.7)$ & $80(66.1)$ & $30(24.8)$ & $17(12.8)$ & & Reference \\
\hline Unsure, $n(\%)$ & $90(19.2)$ & $13(14)$ & $15(12.4)$ & $24(19.8)$ & 38 (28.6) & & \\
\hline \multicolumn{8}{|c|}{ Pregnant women should not get vaccinated } \\
\hline Agree, $n(\%)$ & $54(11.5)$ & $15(16.1)$ & $7(5.8)$ & $12(9.9)$ & $20(15)$ & \multirow[t]{3}{*}{0.051} & $0.301(0.168,0.538)$ \\
\hline Disagree, $n(\%)$ & $226(48.3)$ & $42(45.2)$ & 89 (73.6) & $61(50.4)$ & $34(25.6)$ & & Reference \\
\hline Unsure, $n(\%)$ & $188(40.2)$ & $36(38.7)$ & $25(20.7)$ & $48(39.7)$ & $79(59.4)$ & & \\
\hline \multicolumn{8}{|c|}{ People who had the flu don't need to get vaccinated } \\
\hline Agree, $n(\%)$ & $4(0.9)$ & $1(1.1)$ & $1(0.8)$ & $1(0.8)$ & $1(0.8)$ & \multirow[t]{3}{*}{0.995} & $1.534(0.158,14.87)$ \\
\hline Disagree, $n(\%)$ & $439(93.8)$ & $88(94.6)$ & $117(96.7)$ & $113(93.4)$ & $121(91)$ & & Reference \\
\hline Unsure, $n(\%)$ & $25(5.3)$ & $4(4.3)$ & $3(2.5)$ & $7(5.8)$ & $11(8.3)$ & & \\
\hline
\end{tabular}

${ }^{a} p$-value for association between survey item and profession

Table 4 Perceived personal risk $(N=468)$

\begin{tabular}{|c|c|c|c|c|c|c|c|}
\hline & All & Nurses & Doctors & Allied health professionals & Others & $p$-value ${ }^{a}$ & OR for vaccination (95\% Cl) \\
\hline \multicolumn{8}{|l|}{ I catch the flu easily } \\
\hline Agree, $n(\%)$ & $75(16)$ & $10(10.8)$ & $22(18.2)$ & $19(15.7)$ & $24(18)$ & \multirow[t]{3}{*}{0.431} & $1.613(0.921,2.824)$ \\
\hline Disagree, $n(\%)$ & $292(62.4)$ & $59(63.4)$ & $78(64.5)$ & $80(66.1)$ & $75(56.4)$ & & Reference \\
\hline Unsure, $n(\%)$ & $101(21.6)$ & $24(25.8)$ & $21(17.4)$ & $22(18.2)$ & $34(25.6)$ & & \\
\hline \multicolumn{8}{|c|}{ If I don't get vaccinated I will get sick with the flu } \\
\hline Agree, $n(\%)$ & $144(30.8)$ & $28(30.1)$ & $47(38.8)$ & $35(28.9)$ & $34(25.6)$ & \multirow[t]{3}{*}{0.132} & $3.526(2.158,5.761)$ \\
\hline Disagree, $n(\%)$ & $189(40.4)$ & $35(37.6)$ & $44(36.4)$ & $58(47.9)$ & $52(39.1)$ & & Reference \\
\hline Unsure, $n(\%)$ & $135(28.8)$ & $30(32.3)$ & $30(24.8)$ & $28(23.1)$ & $47(35.3)$ & & \\
\hline \multicolumn{8}{|c|}{ If I get sick with the flu I may infect my family } \\
\hline Agree, $n(\%)$ & $446(95.3)$ & $91(97.8)$ & $118(97.5)$ & $115(95)$ & $122(91.7)$ & \multirow[t]{3}{*}{0.09} & $7.355(2.66,20.333)$ \\
\hline Disagree, $n(\%)$ & $10(2.1)$ & $1(1.1)$ & $3(2.5)$ & $3(2.5)$ & $3(2.3)$ & & Reference \\
\hline Unsure, $n(\%)$ & $12(2.6)$ & $1(1.1)$ & & $3(2.5)$ & $8(6)$ & & \\
\hline \multicolumn{8}{|c|}{ If I get sick with the flu I may infect my patients } \\
\hline Agree, $n(\%)$ & $431(92.1)$ & $89(95.7)$ & $118(97.5)$ & $107(88.4)$ & $117(88)$ & \multirow[t]{3}{*}{0.008} & $4.092(2.022,8.282)$ \\
\hline Disagree, $n(\%)$ & $15(3.2)$ & $2(2.2)$ & & $7(5.8)$ & $6(4.5)$ & & Reference \\
\hline Unsure, $n(\%)$ & $22(4.7)$ & $2(2.2)$ & $3(2.5)$ & $7(5.8)$ & $10(7.5)$ & & \\
\hline
\end{tabular}

${ }^{a} p$-value for association between survey item and profession 
infect patients was slightly lower (92\%); agreement was more common among doctors (98\%) and nurses (96\%) than among allied health professionals (88\%). The OR for vaccination was 7.4 (95 \% CI 2.7, 20.3) for participants who agreed that if they get sick with influenza they may infect their family, higher compared to the OR for vaccination $(4.1,95 \%$ CI 2, 8.3) for participants who agreed that if they get sick with influenza they may infect patients.

\section{Practices of recommending vaccination and attitude towards mandatory vaccination}

Practices regarding recommending vaccination are presented in Table 5: 322 (69\%) recommended vaccination to family, and 210 (77 \% of participants engaged in direct patient care) indicated that they recommended vaccination to patients. Of the participants who were vaccinated, $86 \%$ recommended vaccination to family and $86 \%$ recommended vaccination to patients, as compared to 35 and $54 \%$, respectively, in participants who were unvaccinated.

Only 249 (53\%) of participants were in favor of a mandatory vaccination policy: $65 \%$ among vaccinated participants, and $30 \%$ among unvaccinated participants.

\section{Reasons for vaccine refusal and vaccine side effects}

Reasons for vaccine refusal in unvaccinated participants were: fear of needles (19\%); fear of side effects (66\%); lack of time (16\%); and medical exemption (4\%). Fear of side effects was the most frequent reason for all professions. Fear of needles was lower among doctors and nurses than other professions. Lack of time was more frequent for doctors than for other professions.

Among vaccinated participants, $27 \%$ indicated they had side effects after receiving the vaccine; $38 \%$ of nurses, $22 \%$ of doctors, $26 \%$ of allied health professionals, and $25 \%$ of others.

\section{Multivariate analysis}

Survey items that were independently associated with vaccination in the multivariate logistic regression model were: "The most effective way to prevent influenza is vaccination" (OR 4.0795 \% CI 2.51, 6.58); "Hospital workers are at increased risk of contracting influenza because of their job" (OR $2.8295 \%$ CI 1.56, 5.13); "Vaccine can cause the flu" (OR $0.4195 \%$ CI 0.25, 0.65); "If I don't get vaccinated I will get sick with the flu" (OR $1.9695 \%$ CI 1.12, 3.42); and "If I get sick with the flu I may infect my family" (OR 4.5495 \% CI 1.38, 14.97). The survey item "If I get sick with the flu I may infect my patients" was not independently associated with vaccine uptake. Furthermore, gender, age, ward and profession were not significant predictors of vaccination.

\section{Discussion}

Influenza vaccination coverage at our hospital is unsatisfactory: $42 \%$ of all HCP. However, our vaccination coverage was consistent with the national average [9]. This study revealed that there are misconceptions and significant gaps in knowledge of the risk of influenza and of the influenza vaccine among HCP at our hospital, despite extensive efforts to educate HCP during the influenza vaccination campaign. HCPs' knowledge of influenza vaccine has been directly correlated with vaccination uptake $[10,11]$. In a meta-analysis of studies sponsored by the US Centers for Disease Control and Prevention, HCPs' misconceptions about influenza vaccination were similar to those held by the general public [12]. Similarly, in a study by Gesser-Edelsburg et al. concerning Israeli HCP's attitudes towards the avian influenza vaccine in an outbreak setting, there was no difference between $\mathrm{HCP}$ and the general public regarding support for vaccination [13].

In a large survey of HCP in 2 US medical centers, the most frequent barriers to vaccination against influenza cited by participants who declined vaccination were fear

Table 5 Practices regarding recommending vaccination $(N=468)$

\begin{tabular}{|c|c|c|c|c|c|c|}
\hline & All & Nurses & Doctors & Allied health professionals & Others & $p$-value ${ }^{b}$ \\
\hline \multicolumn{7}{|c|}{ Did you recommend vaccination to family? } \\
\hline Yes, $n(\%)$ & $322(68.8)$ & $61(65.6)$ & $105(86.8)$ & $75(62)$ & $81(60.9)$ & \multirow[t]{2}{*}{$<0.001$} \\
\hline No, $n(\%)$ & $146(31.2)$ & $32(34.4)$ & $16(13.2)$ & $46(38)$ & $52(39.1)$ & \\
\hline \multicolumn{7}{|c|}{ Did you recommend vaccination to patients? } \\
\hline Yes, $n(\%)$ & $210(44.9)$ & $58(62.4)$ & $101(83.5)$ & $26(21.5)$ & $25(18.8)$ & \multirow[t]{3}{*}{$<0.001$} \\
\hline No, $n(\%)$ & $64(13.7)$ & $13(14)$ & $9(7.4)$ & $29(24)$ & $13(9.8)$ & \\
\hline$N R^{a}, n(\%)$ & $194(41.5)$ & $22(23.7)$ & $11(9.1)$ & $66(54.5)$ & $95(71.4)$ & \\
\hline \multicolumn{7}{|c|}{ Are you in favor of mandatory vaccination policy for hospital workers? } \\
\hline Yes, $n(\%)$ & $249(53.2)$ & $52(55.9)$ & $68(56.2)$ & $57(47.1)$ & $72(54.1)$ & \multirow[t]{2}{*}{0.464} \\
\hline No, $n(\%)$ & 219 (46.8) & $41(44.1)$ & $53(43.8)$ & $64(52.9)$ & 61 (45.9) & \\
\hline
\end{tabular}

${ }^{a} N R$ Not relevant

${ }^{\mathrm{b}} p$-value for association between survey item and profession 
of side effects (39\%) and fear of contracting influenza from the vaccine (25\%) [14]. In our study, almost half of participants agreed that you can get sick with influenza from being vaccinated and only $58 \%$ disagreed that vaccine side effects are worse than influenza infections. Among participants who declined vaccination, fear of side effects was the most frequent reason (66 \%), consistent with previous studies [15].

Our study also points to significant differences in knowledge and attitudes between healthcare professions. The proportion of doctors who agreed that vaccination was the best way to prevent influenza was higher as compared to nurses and allied health professionals. Similarly, the proportion of HCP who perceived the vaccine to be harmful was higher among nurses and allied health professionals than among doctors. Similar differences between physicians and nurses have been reported in previous studies [16]. This may explain the lower vaccination rates of nurses and allied health professionals as compared to doctors seen in our study and also at the national level [9].

Other barriers to vaccination reported in previous studies include a low perceived vaccine efficacy and a low perceived risk of influenza infection [14]. In our study, only $62 \%$ agreed that vaccination is the most effective way to prevent influenza, and only $16 \%$ agreed that they get infected with influenza easily. In an analysis of HCP vaccination decisions, skewed risk perceptions concerning disease, transmission and vaccine side effects affected vaccination decisions. When the perceived risk of becoming infected is low, fear of side effects is the most frequent reason to decline vaccination [17]. In our study, most participants perceived themselves to be at low risk of infection with influenza, while a high proportion expressed concern over vaccine side effects.

In the multivariate analysis independent predictors of vaccination were perceived increased risk to self and family, and belief that vaccine is effective, while fear that the vaccine can cause disease was a barrier to vaccination. The belief that HCP might transmit influenza to patients was not independently associated with vaccine uptake. HCP are expected to be highly motivated to protect their patients. However, a review of studies on attitudes and predictors of $\mathrm{HCP}$ vaccination concluded that HCP get vaccinated primarily for their own benefit and not for the benefit of their patients [18]. A systematic review of factors associated with HCP acceptance of vaccination found that desire for self-protection and desire to protect family and friends increased acceptance, while concern that the vaccine causes the illness it was meant to prevent decreased acceptance [19].

In our study, almost all participants agreed that the risk of influenza transmission to patients is high. However, between these two motivators - risk to self and risk to patients - personal risk is the more powerful motivator of vaccine uptake.

Our study also showed that HCP who were vaccinated were more likely to recommend vaccination to patients and family.

Our study has several limitations. Volunteer bias may be present: $66 \%$ of study participants indicated they were vaccinated as compared with the $42 \%$ actual $\mathrm{HCP}$ vaccination rate, thus study participants represent $\mathrm{HCP}$ more inclined to vaccination. Since the survey was conducted after the influenza campaign, beliefs and attitudes may have been biased by behavior. There was also overrepresentation of allied health professionals. Since the study was anonymous, actual vaccination status of study participants could not be verified.

\section{Conclusions}

Our findings suggest that HCP decline vaccination because they do not perceive a personal risk of influenza infection and are concerned about side effects. Preventing influenza transmission from HCP to patients, while most important from an administrative point of view, was not a powerful motivator. Thus, in order to increase vaccine coverage, it is important to continue educating $\mathrm{HCP}$ to correct misconceptions concerning vaccine safety and effectiveness while promoting the benefit of getting vaccinated in order to protect themselves and their family. We plan to use these findings in our influenza vaccination program and target populations with low vaccination rates for more intense intervention.

\section{Funding}

No funding was received for this research.

\section{Authors' contributions}

AN conceived the study, participated in its design and coordination, performed the statistical analysis and drafted the manuscript. NY participated in its design and coordination, and helped draft the manuscript. All authors read and approved the final manuscript.

\section{Authors' information}

Amir Nutman, MD, MPH, is a fellow in healthcare administration and medical assistant to CEO, Tel-Aviv Sourasky Medical Center. He specialized in Internal Medicine and serves as epidemiology consultant to the Occupational Health Clinic at Tel-Aviv Sourasky Medical Center.

Naomi Yoeli, M.A., M. Ed, is a senior clinical and medical psychologist,

organization consultant to management, Tel Aviv Sourasky Medical Center.

\section{Competing interests}

The authors declare that they have no competing interests.

\section{Ethics approval and consent to participate}

The protocol was approved by the Tel-Aviv Sourasky Medical Center Institutional Review Board.

Received: 25 January 2016 Accepted: 11 October 2016

Published online: 26 October 2016

\section{References}

1. World Health Organization. WHO position paper on influenza vaccines November 2012. In: Weekly Epidemiological Record (WER), vol. 47. Geneva: World Health Organization; 2012. p. 461-76. 
2. Ahmed F, Lindley MC, Allred N, Weinbaum CM, Grohskopf L. Effect of influenza vaccination of healthcare personnel on morbidity and mortality among patients: systematic review and grading of evidence. Clin Infect Dis. 2014;58:50-7.

3. Wilde JA, McMillan JA, Serwint J, Butta J, O'Riordan MA, Steinhoff MC. Effectiveness of influenza vaccine in health care professionals: a randomized trial. JAMA. 1999;281:908-13.

4. Kassianos G. Willingness of European healthcare workers to undergo vaccination against seasonal influenza: current situation and suggestions for improvement. Drugs Context. 2015;4:212268.

5. Heinrich-Morrison K, McLellan S, McGinnes U, Carroll B, Watson K, Bass P, Worth $L$, Cheng AC. An effective strategy for influenza vaccination of healthcare workers in Australia: experience at a large health service without a mandatory policy. BMC Infect Dis. 2015;15:42

6. Pitts SI, Maruthur NM, Millar KR, Perl TM, Segal J. A systematic review of mandatory influenza vaccination in healthcare personnel. Am J Prev Med. 2014:47:330-40.

7. Ministry of Health Israel. Director General's Communication: Vaccination of Health Care Workers; 2013. Available at: https://www.health.gov.l/hozer/ mk07_2013.pdf.

8. Habib S, Rishpon S, Rubin L. Influenza vaccination among healthcare workers. Isr Med Assoc J. 2000;2:899-901.

9. Ministry of Health Israel. Vaccination of Medical Teams for Influenza, Winter 2014/5. 2015. Available at: https://www.health.gov.l//PublicationsFiles/Flu_ stuff2014_2015.pdf.

10. Martinello RA, Jones L, Topal JE. Correlation between healthcare workers' knowledge of influenza vaccine and vaccine receipt. Infect Control Hosp Epidemiol. 2003;24:845-7.

11. Nativ T, Paz A, Peterfreund I, Potasman I. Influence of knowledge and attitude on the uptake of influenza vaccine by healthcare workers. Harefuah. 2010;149:626-9. 685.

12. Nowak GJ, Sheedy K, Bursey K, Smith TM, Basket M. Promoting influenza vaccination: insights from a qualitative meta-analysis of 14 years of influenza-related communications research by U.S. Centers for Disease Control and Prevention (CDC). Vaccine. 2015;33:2741-56.

13. Gesser-Edelsburg A, Walter N, Green MS. Health care workers-part of the system or part of the public? Ambivalent risk perception in health care workers. Am J Infect Control. 2014:42:829-33.

14. Naleway AL, Henkle EM, Ball S, Bozeman S, Gaglani MJ, Kennedy ED, Thompson MG. Barriers and facilitators to influenza vaccination and vaccine coverage in a cohort of health care personnel. Am J Infect Control. 2014;42:371-5.

15. Awali RA, Samuel PS, Marwaha B, Ahmad N, Gupta P, Kumar V, Ellsworth J, Flanagan E, Upfal M, Russell J, et al. Understanding health care personnel's attitudes toward mandatory influenza vaccination. Am J Infect Control. 2014:42:649-52.

16. Jaiyeoba O, Villers M, Soper DE, Korte J, Salgado CD. Association between health care workers' knowledge of influenza vaccine and vaccine uptake. Am J Infect Control. 2014;42:69-70.

17. Betsch C. Overcoming healthcare workers vaccine refusal-competition between egoism and altruism. Euro Surveill. 2014;19:20979.

18. Hollmeyer HG, Hayden F, Poland G, Buchholz U. Influenza vaccination of health care workers in hospitals-a review of studies on attitudes and predictors. Vaccine. 2009;27:3935-44.

19. Vasilevska M, Ku J, Fisman DN. Factors associated with healthcare worker acceptance of vaccination: a systematic review and meta-analysis. Infect Control Hosp Epidemiol. 2014;35:699-708.

\section{Submit your next manuscript to BioMed Central and we will help you at every step:}

- We accept pre-submission inquiries

- Our selector tool helps you to find the most relevant journal

- We provide round the clock customer support

- Convenient online submission

- Thorough peer review

- Inclusion in PubMed and all major indexing services

- Maximum visibility for your research

Submit your manuscript at www.biomedcentral.com/submit

CBiomed Central 